Anuario Latinoamericano Ciencias Políticas

y Relaciones Internacionales

vol. 1, 2014

p. $71-88$

\title{
La emergencia de los BRICS: Brasil y Sudáfrica en las relaciones Sur-Sur
}

\author{
Carlos Alfredo da Silva \\ UNIVERSIDAD NACIONAL DE ROSARIO, \\ ARGENTINA \\ $\triangle$ gadatipe@cablenet.com.ar
}

\begin{abstract}
RESUMEN
El presente trabajo tiene por propósito reflexionar críticamente sobre los desafíos y dilemas que se presentan a Brasil y Sudáfrica, países emergentes denominados con el acrónimo BRICS, en las relaciones Sur-Sur. Asimismo, se ponderará el rol de Brasil y Sudáfrica, como miembros activos del grupo BRICS (Brasil, Rusia, India, China y Sudáfrica). Finalmente, se evaluarán las posibilidades de inserción y relacionamiento externo de ambos Estados emergentes (Brasil y Sudáfrica), en función de los intereses y relaciones internacionales brasileños, en un contexto de creciente asimetría regional. Se utilizará un enfoque metodológico cualitativo, desde la perspectiva de la historia de las relaciones internacionales contemporáneas.
\end{abstract}

PALABRAS CLAVE: Brasil, BRICS, relaciones Sur-Sur, Sudáfrica

\section{SUMMARY}

The purpose of this paper is to critically reflect on the challenges and dilemmas that are faced by Brazil and South Africa, as emerging countries denominated with the acronym of BRICS, in the context of South-South relations. We will also analyze the role of both countries, Brazil and South Africa, as active members of the BRICS group (Brazil, Russia, India, China and South Africa). Finally, the potential for integration and external relations of both emerging states (Brazil and South Africa) will be assessed, focusing on the interests and international relations of Brazil, in a context of growing regional asymmetry. A qualitative methodological approach will be used in this paper, from the perspective of the history of international relations.

KEYWORDS: Brazil, BRICS, South-South relations, South Africa. 
América Latina: cambios a nivel regional y en su inserción internacional

\section{Introducción y reflexiones teóricas}

Si bien siempre han existido diferentes vínculos entre los países ubicados en el hemisferio sur, las relaciones entre los mismos han adquirido mayor relevancia desde la Conferencia de Bandung del año 1955. En este contexto, surge la necesidad de dichos países de establecer reglas y principios propios de acción frente al comportamiento de las superpotencias en la guerra fría: los Estados Unidos de América y la Unión de Repúblicas Socialistas Soviéticas.

Tras la independencia de algunos Estados africanos y asiáticos, sus líderes convocaron la Conferencia que se llevó a cabo en Bandung (Indonesia), cuyo objetivo principal fue el fortalecimiento de las relaciones entre estos países, a la vez que procuraban favorecer la cooperación en diversos ámbitos. Paralelamente, se acordaron una serie de principios que regirían sus relaciones exteriores, vinculados con el anticolonialismo, el no alineamiento y la afirmación de la independencia (Zorgbibe 1997).

Las relaciones internacionales entre estos países descolonizados han sido definidas dentro del marco institucional de la mencionada Conferencia de Bandung, viéndose reflejadas en la constitución del movimiento de países no alineados (MPNOAL), proyecto político que se vio suspendido con el triunfo y aplicación del neoliberalismo y del Consenso de Washington en Europa y Estados Unidos, luego diseminado hacia el "resto del mundo".

A pesar del impulso inicial de estos países por lograr un acercamiento y una posición compartida en las relaciones internacionales, hay que tener en cuenta que es recién en la posguerra fría cuando estos intentos vuelven a recobrar importancia, buscando profundizar las relaciones Sur-Sur.

En la actualidad, los cambios en el sistema internacional transforman las funciones de los Estados nacionales y del poder. La globalización y la relación directa entre lo local y lo global llevan a enfrentar a los Estados con nuevas problemáticas y amenazas, como el cambio climático, el terrorismo, la escasez de recursos energéticos, entre otros. Por tal motivo, observamos que la interdependencia que resulta de las relaciones entre los actores estatales y la complejidad del sistema internacional requiere de una mayor integración y cooperación con respuestas y responsabilidades conjuntas.

En este contexto, es necesario fomentar el multilateralismo para poder negociar con los países más poderosos, y porque los flujos transnacionales requieren más cooperación e integración entre los gobiernos involucrados (Sousa de 2008: 1-14).

Asimismo, el proceso de globalización y creciente interconexión transnacional entre gobiernos y actores no estatales está cambiando el rol del Estado. La globalización ha disminuido este papel, pero, a la vez, hay una revalorización del Estado como actor que negocia, acelera, promociona y gestiona la relación con otros actores globales. En este sentido, el factor nacionalista, que en mayor o menor medida está presente en todas las potencias emergentes, hace que el Estado desempeñe una función fundamental (de Sousa 2008: 2). 
Con el objetivo de analizar los desafíos que se presentan a Brasil y Sudáfrica como países emergentes en las relaciones Sur-Sur, se torna relevante considerar la creciente importancia que han ido adquiriendo algunos países del sur, caracterizados por los analistas como los principales motores del crecimiento y desarrollo en las décadas venideras.

Brasil es uno de los países que conforman el grupo BRIC (Brasil, Rusia, India y China), marca con impacto mediático. Este acrónimo fue utilizado por primera vez en el año 2001 por Jim O'Neill (jefe de investigaciones económicas globales) de Goldman Sachs, ${ }^{1}$ el cual considera que Brasil, Rusia, India y China serán las principales economías hacia la década de 2050, debido a que durante la década de 2000 el Producto Bruto Interno de estos cuatro países ha crecido a tasas superiores al PBI de los países del $\mathrm{G}^{2}$, llegando a representar aproximadamente el $23 \%$ del producto mundial (O’Neill 2001). Estos países, teniendo en cuenta sus proyecciones demográficas, los niveles de acumulación de capital y su productividad, serán las cuatro economías con mayor peso en la economía mundial hacia el año 2050 (Wilson 2003).

Actualmente, los países miembros del BRIC abarcan el 25\% de la superficie terrestre, poseen más del $41 \%$ de la población mundial con 2.882 millones de habitantes y representaron el 18\% de la economía global en el año 2010. Por otra parte, las relaciones comerciales entre estas potencias emergentes se multiplicaron por 15 de 2001 a 2010, estimándose en 230 mil millones de dólares.

Estos países han pasado a ser considerados como candidatos a jugar un rol destacado en el escenario mundial. "Grandes mercados internos aumentan las posibilidades de que se puedan obtener 'exportaciones viabilizadas por el crecimiento', más que un 'crecimiento liderado por las exportaciones', lo que implica mayores espacios para un rol activo en las relaciones internacionales" (Baumann 2009: 2). Los más de 2.882 millones de habitantes de los países que componen el BRICS tendrán un PBI de casi 10 billones de dólares y sus fuentes de ingresos son muy diversas. China es el principal exportador mundial de productos manufacturados y pronto lo será de tecnología. India consigue sus recursos principalmente por servicios, básicamente relacionados con software. Brasil basa su economía en la exportación de materias primas agrícolas y próximamente ocupará una posición de liderazgo en biodiversidad. Rusia basa su desarrollo en la exportación de minerales, fundamentalmente petróleo y gas natural, la tecnología relacionada con la explotación de estas materias primas, y en la exportación de tecnología para la construcción de infraestructura de transporte (trenes, camiones, autobuses y aviones), área en la cual tiene una amplia trayectoria y reconocimiento mundial, sin olvidar las telecomunicaciones y lo relativo a satélites.

\footnotetext{
1 Goldman Sachs, grupo de inversión dedicado a la banca de inversión, gestión de inversiones y servicios financieros a clientes institucionales. Fue fundado en 1869.

2 El G7 es el grupo de países más industrializados que surgió en 1973 (Alemania, Canadá, Estados Unidos, Francia, Italia, Japón y Gran Bretaña). En el año 1998 se incorporó Rusia y pasó a conocerse como el G8.
}

La emergencia de los BRICS: Brasil y Sudáfrica en las relaciones Sur-Sur

Carlos Alfredo da Silva 
América Latina: cambios a nivel regional y en su inserción internacional
En una conferencia celebrada en China durante el mes de Abril de 2011, los líderes del grupo BRIC invitaron a participar por vez primera a la República de Sudáfrica, con el fin de analizar diversas cuestiones internacionales, hablándose ahora de BRICS: Brasil, Rusia, India, China y Sudáfrica. El documento de Goldman Sachs reconoce que Sudáfrica hacia el año 2050 tendrá un PIB menor que los demás, sin embargo, se reconoce que tendrá un nivel más alto de renta per cápita. Este y otros indicadores han llevado a que se incluya Sudáfrica en ese grupo.

En dicha cumbre participaron los presidentes: de Brasil, Dilma Rousseff; de Rusia, Dimitri Medvédev; de China, Hu Jintao; de Sudáfrica, Jacob Zuma; además del primer ministro de la India, Manmohan Singh. Los mandatarios coincidieron en la profundización de los intercambios y la cooperación en la industria, el comercio, la ciencia, la tecnología y la agricultura como una necesidad. Por tal motivo, la declaración que resultó de esta cumbre destacó el fortalecimiento de la alianza entre los países emergentes que permitiría tener una postura conjunta en el escenario internacional. Es importante destacar la incorporación oficial de Sudáfrica uniéndose al grupo como miembro de derecho pleno luego de esta cumbre.

Dicha declaración se orientó a temas financieros y económicos, en gran medida, pero también determinó la postura del Foro frente a los conflictos sucedidos en África a principios de 2011, junto con la necesidad de reformar la Organización de Naciones Unidas y el Consejo de Seguridad. Uno de los temas planteados por las potencias emergentes, en dicha reunión, fue la preocupación por los flujos de capital que están empujando a la revalorización de sus monedas y la volatilidad de los precios de las materias primas, producto de las actuales crisis internacionales, que amenaza la recuperación mundial. Además, se destacó la necesidad de cooperación en materia económica ya que el masivo arribo de capitales valora en exceso la moneda de estas economías emergentes, incrementado la presión inflacionaria y afectando la competitividad de sus exportaciones en países con cambio flotante. Por otra parte, los mandatarios manifestaron el apoyo a la reforma y la mejora del sistema monetario internacional. Paralelamente, estos países se comprometieron a brindar líneas de crédito en monedas nacionales para reducir la dependencia del dólar e incrementar las inversiones entre los miembros del BRICS y la participación de empresas medias.

La presidenta brasileña, Dilma Rousseff, indicó durante la rueda de prensa que los países del BRICS reforzarán aún más la coordinación y aumentarán el diálogo. Rousseff destacó que los 5 países han dado muestras de un gran potencial en su lucha contra la crisis financiera y afirmó que el bloque está desempeñando un papel cada vez más importante en el ámbito de la economía y la política3. Marcó el punto central al expresar "estamos encargados de crear un mundo institucional multipolar sin hegemonías".

3 Dilma Rouseff, Conferencia de prensa BRICS, 2010, http://espanol.cntv.cn/espanol/special/brics/portada/index.shtml, consulta: 24.05.2011. 
Los temas de la agenda del grupo BRICS que se destacan son: la crisis económica mundial, la reforma de la Organización de Naciones Unidas (ONU), nuevo enfoque del comercio global con reglas más transparentes y cambios en las instituciones financieras internacionales con el fin de dar mayor participación a los países emergentes.

A los fines de esta investigación se tendrá en cuenta principalmente las relaciones entre Brasil y Sudáfrica en el marco del grupo BRICS, considerándolos como los países con mayor proyección internacional de América Latina y África, respectivamente.

\section{Brasil, su política exterior bajo los gobiernos de Lula y Rousseff}

Como nos plantea la especialista Miriam Gomes Saraiva (2010: 69-71), la política exterior brasileña se caracteriza por la continuidad y, por otro lado, algunas creencias que orientan su evolución desde hace muchos años: la autonomía, la acción universalista y la idea de que el país ocupará un lugar de mayor preponderancia en la política internacional. La fuerte concentración del proceso de formulación de la política exterior puede orientarse hacia una estrategia de carácter multipolar o de búsqueda de ganancias relativas en el escenario internacional; hacia una preferencia por una actuación más autónoma o por liderar las iniciativas de los países del Sur; hacia un comportamiento del país como stakeholder o como revisionista soft. En Itamaraty, hay básicamente dos corrientes de pensamiento con percepciones diferentes sobre las estrategias y alianzas externas. Los institucionalistas pragmáticos, predominantes durante el gobierno de F. H. Cardoso, se caracterizan por dar mayor importancia al apoyo de Brasil a los regímenes internacionales en vigencia. Esta postura defiende una mayor identificación del país con Occidente como un escenario favorable al desarrollo económico brasileño. La corriente autonomista, que se consolidó como principal formuladora de la política exterior durante el gobierno de Lula, busca una proyección más autónoma y proactiva de Brasil en la política internacional. Los autonomistas defienden una reforma de la dinámica de las instituciones multilaterales, en el sentido de crear espacios de acción para el país y asumir así un perfil revisionista del orden internacional. La construcción del liderazgo regional, de un liderazgo entre los países del Sur, y el ascenso a la posición de potencia global son sus objetivos principales. En el campo económico, buscan una estrategia de inserción internacional orientada al intercambio tecnológico y a la proyección de las empresas brasileñas. Durante el gobierno de Lula, este grupo estableció un diálogo importante con Itamaraty a través de la figura del Presidente, que tuvo un fuerte desempeño en el campo de la diplomacia. Para estos pensadores, la integración regional con base en una identidad sudamericana sería vista como una prioridad de la política exterior. Esta composición produjo una discontinuidad en la visión del mundo y en las estrategias adoptadas por
La emergencia de los BRICS: Brasil y Sudáfrica en las relaciones Sur-Sur

Carlos Alfredo da Silva 
América Latina: cambios a nivel regional y en su inserción internacional la diplomacia brasileña y llevó al país a un movimiento de consolidación de su presencia internacional en el rol de global player.

Brasil tiene un potencial para sembrar más de 100 millones de hectáreas en la zona del "cerrado" que cada vez es más abierta a las producciones. Su sector agroindustrial genera exportaciones por encima del $43 \%$ de lo que su economía produce: $26 \%$ son exportaciones de soja, $19 \%$ de carne (Brasil es el primer exportador de carne en el mundo), $16 \%$ de etanol (es el cuarto productor mundial de biocombustibles).

Uno de los ejes del nuevo gobierno de la Presidente Dilma Rousseff es el énfasis en los derechos humanos, algo que genera profundos cambios en política interior y exterior. Esta mujer, guerrillera marxista que padeció la represión y tortura de la dictadura (1964-1985), no podía soslayar su pasado. Brasil pasa de la política del autoindulto y olvido al de la memoria, verdad y justicia. A partir de incorporar tratados internacionales, como la aprobación por parte del Senado de la Convención Interamericana sobre Desaparecidos, los delitos de la dictadura se encuadran en la figura de los crímenes de lesa humanidad, por lo que la Justicia avanzará sobre sus acciones.

Por otra parte, según la Fundación Getúlio Vargas, Brasil alcanzó su nivel más bajo de desigualdad social en los últimos veinte años y, a la par, se consolida una emergente clase media. La noción de Bel-India, acuñada por Edmar Bacha, para referirse a la estructura desigual, donde socioeconómicamente en los años setenta una pequeña minoría vivía como en Bélgica y una gran mayoría con niveles de pobreza de la India, cuarenta años después deja de representar a Brasil. La tendencia da como resultado la formación de una incipiente clase media, como en Argentina.

Ahora bien, tal como hemos afirmado anteriormente, Brasil ha logrado en veinte años lo que otros países latinoamericanos no han podido, lo cual lo condujo a formular una política dirigida a obtener no sólo el rol de potencia regional en América del Sur, sino también que aspira a convertirse en un actor global relevante del sistema internacional.

En este marco, tanto Cardoso como Lula da Silva han mantenido una continuidad en los cuatro ejes centrales de la política exterior brasileña:

- El reconocimiento como par, en un orden multipolar, por parte de las otras potencias establecidas: China, EEUU, India, Rusia, Sudáfrica y la Unión Europea.

- La aceptación de su liderazgo regional en América del Sur, liderazgo en constante construcción sustentado en la UNASUR y los esquemas de integración y cooperación regional.

- Su participación en la toma de decisiones en los organismos internacionales de mayor relevancia, tal como se ha manifestado en la Organización Mundial de Comercio (OMC), en Naciones Unidas, el Foro de Davos y otros similares.

- Alcanzar la condición de miembro permanente en el Consejo de Seguridad de la Organización de las Naciones Unidas (Grabendorff 2010). 
La política exterior del gobierno de Lula se distinguió, en relación con el período anterior, en términos económicos. Su gestión fue introduciendo progresivamente elementos del desarrollismo, como las iniciativas para el esfuerzo de la infraestructura y un proyecto de fortalecimiento de la industrialización, con perspectivas de avances tecnológicos en algunas áreas. Externamente, emprendió una intensa política de búsqueda de mercados para las exportaciones del país, dando prioridad a aliados emergentes y a la exportación de bienes completos, así como a acuerdos de cooperación tecnológica de diferentes matices. El ascenso de la corriente autonomista disminuyó la adhesión a los regímenes internacionales, que fue sustituida por un comportamiento activo con vistas a modificarlos a favor de los países del Sur o en beneficio propio. La idea de atraer a otros países del Sur, emergentes o de menos recursos, sirvió de base para la actuación internacional del país. El liderazgo regional en América del Sur se convirtió en un objetivo y, aún más, en un deseo político de la presidencia. La construcción de este liderazgo regional se apoyó en una articulación entre los autonomistas de Itamaraty y el grupo vinculado con el PT. La aproximación con los países vecinos es percibida por los autonomistas como un instrumento para una mejor inserción internacional, que posibilita la realización del potencial brasileño y la formación de un bloque capaz de ejercer mayor influencia internacional. Sería importante que Brasil asumiera el papel de paymaster del proceso de integración en la región y frente a países vecinos con gobiernos antiliberales. El resultado fue un aumento progresivo del rol de paymaster de la diplomacia brasileña, junto con una búsqueda de construcción de consensos políticos entre sus pares frente a temas que afectan a la región. Se dio un nuevo peso a la construcción de un liderazgo brasileño en la región con patrones basados en el refuerzo del multilateralismo (con énfasis en UNASUR). Actualizó con principios de la no intervención en la forma de la "no indiferencia" y vinculó iniciativas de cooperación e integración regional con incentivos al desarrollo brasileño. El Mercosur dejó de tener un papel importante en la estrategia brasileña global y pudo ser visto dentro de la perspectiva sudamericana.

El gobierno de Dilma Russeff parece representar el mantenimiento de las estrategias de política externa del gobierno anterior: la trayectoria revisionista frente a las instituciones internacionales, la actuación como representante de los países del Sur y el liderazgo regional. Los autonomistas mantuvieron su predominio dentro de Itamaraty, aunque dando lugar a generaciones más jóvenes, con una visión del mundo más globalizada. Ya comenzó a mostrar inflexiones en relación con el proceso de formulación de la política exterior y los ajustes en su aplicación.

Compartiendo lo expresado por el especialista Iole Ilíadas Lopes (2010: 63-68), Dilma Rousseff representa a aquellos que en el pasado fueron detenidos o torturados por luchar por la democratización del país. Vista en su dimensión histórica, esta victoria se inserta en un contexto más amplio de disputa entre dos modelos de desarrollo capitalista en Brasil: la corriente conservadora y la corriente democrática. Esta última ha sido hegemonizada, en
La emergencia de los BRICS: Brasil y Sudáfrica en las relaciones Sur-Sur

Carlos Alfredo da Silva 
América Latina: cambios a nivel regional y en su inserción internacional el período más reciente de la historia del país, por las fuerzas de izquierda, sobre todo por el Partido de los Trabajadores (PT). Eso implica profundizar el carácter democrático y popular del proyecto de desarrollo implementado en el país, ampliando el papel del Estado como inductor del crecimiento económico y realizando reformas estructurales que efectivamente distribuyan riqueza, poder y derechos, tales como la reforma tributaria, la reforma agraria, la reforma urbana, la reforma política y la reforma educativa, además de la democratización de los medios de comunicación, de la consolidación del sistema universal de salud (conocido como SUS) y de la profundización de la integración latinoamericana. La disputa sobre rumbos y proyectos también se manifiesta en el interior del propio gobierno. Es importante señalar que este es un gobierno de coalición, cuya base de apoyo no son solamente los partidos de izquierda. Además de enfrentarse con la oposición de derecha y con las contradicciones internas del gobierno, el tercer desafío del mandato de Dilma Rousseff será lidiar con un cuadro económico mundial inestable y de bajo dinamismo, y con los riesgos crecientes de que el alza de los precios de los commodities genere, por un lado, inflación y, por otro, una creciente primarización de la estructura productiva y de las exportaciones brasileñas. El precio de los combustibles tiende a inviabilizar una política de crecimiento económico con distribución de la renta, sin que se realicen efectivamente reformas estructurales y sin que se toquen los intereses del capital financiero y de los sectores monopolizados de la economía.

\section{República de Sudáfrica}

En Sudáfrica, en el ámbito interno, no existen aún cifras exactas de la cantidad de víctimas que dejó el apartheid. Se calcula que son varios miles. Fue casi un siglo de régimen segregacionista que comenzó en 1910 con el nacimiento de la Unión Sudafricana y que concluyó - al menos en lo institucional - el 27 de abril de 1994, cuando Nelson Rolihlahla Mandela se convirtió en el primer presidente negro tras el triunfo aplastante - el 62\% de los votos - del Congreso Nacional Africano (CNA). Esa histórica elección permitió votar a los 40 millones de sudafricanos - blancos, negros, coloured (mestizos). Desde entonces, se celebra el "Día de la Libertad”, hoy la fiesta patria más importante.

Sudáfrica es relevante por su ubicación geopolítica en el Atlántico Sur y el Índico, su posición negociadora en el comercio internacional agropecuario y por una historia exitosa del siglo XX, por su transición ordenada y pacífica de un régimen político retrógrado, basado en la segregación racial, donde una minoría blanca controla a una mayoría negra, hacia una sociedad democrática. En el plano internacional y regional, éste país tiene, además, una innegable proyección en todo el continente africano, cuya población será de 1.500 millones de personas en el 2030. Su carácter de miembro del grupo BRICS es percibido como algo que potenciará su protagonismo en el desarrollo africa- 
no. Y, a su vez, China ya tiene una especial presencia económica en Sudáfrica. Es el principal socio africano de China, tanto por su intercambio comercial como en inversiones directas.

Concebido como un espacio geopolítico informal que apunta a incidir en la reformulación de los mecanismos formales de gobernabilidad internacional, el BRICS original se enriquece con la incorporación de Sudáfrica, lo cual le otorga una "dimensión africana" que carecía, por ser la mayor economía de la región. A su vez, África está adquiriendo en su conjunto una clara relevancia económica global. Pero la dimensión económica de Sudáfrica es reducida con respecto a los BRICS, tanto en términos de población, de producto bruto interno y de participación en el comercio mundial. Y si se considera su incorporación al grupo informal interregional sólo a partir de criterios económicos, pueden tener razón quienes señalan que no hubiera sido Sudáfrica un candidato ideal. Su producto interno bruto, por ejemplo, es sólo un cuarto que el de Rusia.

La invitación cursada a Sudáfrica en 2009 por el gobierno chino en nombre de los otros países miembros (Brasil, Rusia e India) para incorporarse al grupo y el hecho de que, en abril de 2010, se realizó la tercera Cumbre en Beijing, le puede otorgar a este mecanismo informal de naciones una relevancia especial en el proceso de redefinición de las instituciones para la gobernabilidad económica global. ${ }^{4}$

\section{Intereses brasileños en Sudáfrica}

Históricamente, las relaciones entre América Latina y África han sido de una intensidad relativamente baja, pero en las últimas décadas de la mano de la intensificación de las relaciones Sur-Sur (y dentro de éstas, particularmente las relaciones comerciales Sur-Sur) se puede observar una creciente interrelación entre América Latina y África. ${ }^{5}$ Pero más allá de las relaciones comerciales que se dan entre los países de estos continentes, "hoy en día las relaciones interregionales no se pueden resumir a un aspecto meramente económico" (Brun 2009: 5). Las vinculaciones entre América Latina y África se dan en los aspectos económicos y políticos y en el campo de la cooperación Sur-Sur, pero en diferentes niveles entre los países.

El país latinoamericano que más estrechos vínculos tiene con el continente africano en los aspectos culturales, económicos, políticos y de cooperación es Brasil: es el país que posee la mayor comunidad afrodescendiente; el comercio

4 La decisión de invitar a Sudáfrica habría sido impulsada por China, pero en su anuncio se precisó que se hacía en nombre de los otros miembros. Por lo menos dos, Brasil y Rusia, se apresuraron a darle la bienvenida a Sudáfrica.

5 Se estima que en los últimos años el comercio exterior entre los países del hemisferio sur ha explicado cerca del $40 \%$ del crecimiento económico mundial.
La emergencia de los BRICS: Brasil y Sudáfrica en las relaciones Sur-Sur

Carlos Alfredo da Silva 
América Latina: cambios a nivel regional y en su inserción internacional

exterior con África es uno de los mayores de América Latina; es el país con mayores vinculaciones diplomáticas debido a que es el Estado que posee la mayor cantidad de embajadas en el continente africano; y el que más cantidad de proyectos y programas de cooperación está desarrollando en África, principalmente en el ámbito de la salud pública, en el campo energético y en el sector agroalimentario.

Es necesario subrayar que en los últimos años se observa la presencia de un fenómeno nuevo que consiste en la expansión del comercio entre países en desarrollo, por lo que la relación de los socios comerciales en este tipo de vinculaciones se enmarca dentro de las relaciones Sur-Sur. En este contexto, la fortaleza, el liderazgo, la riqueza y la influencia de Sudáfrica y Brasil, en sus respectivos continentes, son los factores que han desarrollado el fortalecimiento de los vínculos de comercio e inversión entre ellos. Paralelamente, observamos que las ventajas comparativas de los productos de estas economías permiten la expansión, el incremento del comercio y la inversión a nivel regional. Actualmente, Brasil es el mayor socio comercial de Sudáfrica del continente latinoamericano, como resultado de este crecimiento del comercio detallado anteriormente.

Así mismo, con el objetivo de profundizar las relaciones económicas se ha creado el "foro empresarial Brasil-Sudáfrica" que refuerza los lazos comerciales y la inversión entre Brasil y empresas sudafricanas de tecnología de la información, minería, finanzas, infraestructura y el sector farmacéutico. Por otra parte, la Corporación de Desarrollo Industrial de Sudáfrica y el Banco Brasileño de Desarrollo firmaron un acuerdo de cooperación financiera a través de variados proyectos en el sector automotriz, farmacéutico y alimenticio.

Las relaciones de Brasil con Sudáfrica van más allá que el sólo hecho de formar parte del llamado Grupo BRICS. Además de los vínculos en los sectores mencionados precedentemente, se han desarrollado diálogos entre países del hemisferio sur buscando un mejor posicionamiento en el sistema internacional, donde es claramente Brasil el principal interlocutor. Brasil, India y Sudáfrica vienen desarrollando diferentes acciones diplomáticas, dentro de las cuales es importante destacar la puesta en marcha del Foro Trilateral IBSA en el año 2003 mediante la "Declaración de Brasilia" (Declaración de Brasilia 2003). El objetivo de este diálogo es la promoción de la cooperación comercial y diplomática en diversos ámbitos.

IBSA puede ser definida como una asociación formada por tres poderes medios emergentes: India, Brasil y Sudáfrica. La categoría de poder medio emergente surge en el campo de las Relaciones Internacionales como un nuevo subtipo que se distingue dentro del conjunto de las denominadas potencias medias en desarrollo o de segunda generación. ${ }^{6}$ (...) Los países de IBSA

\footnotetext{
6 Durante el periodo de distensión de la Guerra Fría, algunos países medios comenzaron a consolidar áreas de influencia en su región. Esto permite una diferenciación entre las potencias
} 
constituyen claros ejemplos de poderes medios emergentes al contar con un importante liderazgo en cada una de sus regiones sumado a un rol destacado en sucesivas y simultáneas negociaciones globales. (Giaccaglia 2011: 3-4)

Este Foro ha permitido la conexión entre estos tres países en todos los niveles, desarrollándose importantes Cumbres de Jefes de Estado y Reuniones de Ministros de Relaciones Exteriores que han llevado a aumentar la cooperación en diversos ámbitos: coordinación política, cooperación sectorial y promoción de la participación de otros sectores y actores gubernamentales y no gubernamentales.

Pese a las diferencias, la alianza consigue actuar de forma coherente a nivel internacional debido a sus valores y objetivos globales compartidos. Se trata de grandes democracias del Sur, caracterizadas por un pluralismo de culturas, religiones, razas y lenguas. En este sentido, si el Movimiento de Países No Alineados se definía por el antiimperialismo y la exigencia de un nuevo orden internacional, IBSA se define por la democracia y la búsqueda de una mejor posición en el orden existente. (de Sousa 2008: 6)

En el marco de la coordinación política se han establecido posiciones comunes frente a asuntos globales, como por ejemplo en el ámbito de las negociaciones multilaterales en el seno de la OMC. Teniendo en cuenta estas consideraciones, observamos que IBSA actúa como un "grupo de presión internacional gubernamental" con el objeto de influir en las decisiones de los foros y organismos internacionales, ${ }^{7}$ a fin de obtener resultados favorables a sus intereses. Este modelo de coaliciones internacionales implica un acuerdo coordinado frente a las negociaciones multilaterales para lograr un objetivo común. Por esta razón, observamos que este foro actúa con rasgos propios del Tercer Mundo abarcando una multiplicidad de temas como una "coalición de bloque" y, al mismo tiempo, actúa como "coalición por tema" con una meta determinada en función del organismo político en que se encuentre (Giaccaglia 2011). Dentro de la cooperación sectorial se han establecido diferentes grupos de trabajo en sectores específicos que se constituyen en el soporte de la cooperación: ${ }^{8}$ administración pública, agricultura, ciencia y tecnología, comercio, defensa, cultura, energía, educación, salud, medio ambiente y cambio climático, turismo y transporte.

medias tradicionales o de primera generación y las denominadas potencias medias regionales en desarrollo o de segunda generación tales como Brasil, India, Sudáfrica, México, Nigeria, Argentina y Yugoslavia.

7 Tales como la Organización Mundial del Comercio (OMC), Naciones Unidas, la Organización Mundial de la Salud (OMS), entre otros.

8 IBSA, http://www.ibsa-trilateral.org/index.php?option=com_content\&view=article\&id $=97 \&$ Itemid=70, consulta: 24.05 .2011 .
La emergencia de los BRICS: Brasil y Sudáfrica en las relaciones Sur-Sur

Carlos Alfredo da Silva 
América Latina: cambios a nivel regional y en su inserción internacional
En el plano de propiciar la participación de otros actores estatales y de la sociedad civil, se han llevado a cabo diferentes seminarios y foros con representantes de los parlamentos nacionales, sectores académicos, ámbito empresario, entre otros. ${ }^{9}$

En el ámbito específico de la cooperación Sur-Sur, se estableció el "Fondo IBSA para aliviar la pobreza y el hambre", cuyo objetivo es el financiamiento de proyectos de desarrollo en otros países del hemisferio sur. Además de ofrecer trasferencias líquidas de recursos a países de similar nivel de desarrollo, los países del Foro ofrecen sus conocimientos técnicos y científicos en diversas áreas.

El mecanismo de funcionamiento de IBSA implica la reunión de las máximas autoridades, Jefes de Estado y de Gobierno y los cancilleres de los tres países, en diversos encuentros, reflejando una coordinación política del grupo en diversos temas de la agenda internacional. "Desde su creación, desde el punto de vista discursivo, la asociación ha exhortado al establecimiento de un orden internacional predecible, basado en los principios y leyes del Derecho Internacional. Promueve además, una globalización que beneficie a todos los países del mundo y que permita una mayor inclusión y equidad social, así como también el desarrollo de un modelo de cooperación Sur-Sur" (Giaccaglia 2011: 9). Observamos que en pocos años este Foro ha propiciado la profundización de los vínculos entre sus miembros.

El rasgo distintivo de este Foro es la iniciativa de influir en la agenda internacional sin cuestionar la estructura general. La política exterior pretende afrontar los desafíos de la globalización y las transformaciones en el sistema internacional cooperativamente, basándose en el multilateralismo. Observamos que el Foro no prevé, a mediano plazo, una cooperación interregional, pero no será imposible lograr la cooperación trilateral sin incluir a las regiones respectivas. Por ahora cada miembro persigue sus propias políticas a nivel regional (Sousa de 2008).

Tanto Sudáfrica como Brasil podrían clasificarse como potencias emergentes, porque poseen factores básicos que permiten incrementar el crecimiento. Dichos países están encaminados a ser grandes potencias económicas, sobre todo si continúan sus relaciones en el marco del Foro IBSA, intensificando la cooperación Sur-Sur y la coordinación de sus economías para ganar un lugar destacado a nivel internacional. Teniendo en cuenta estas consideraciones, es primordial destacar que estas potencias emergentes cuentan con un potencial demográfico y económico importante, con capacidades que podrían permitir el logro de una mejor posición en el escenario internacional y un liderazgo regional. Es interesante destacar que, a nivel nacional, tanto Brasil como Sudáfrica presentan problemas socioeconómicos internos, viéndose reflejado esto en los índices de distribución del ingreso que muestra la desigualdad socioeconómica presente en ambos países. Según el Índice de Desarrollo $\mathrm{Hu}$ -

9 Seminarios de Desarrollo Social, Foro de Empresarios, Foro de la Mujer, Foro de Parlamentarios, seminarios académicos, Foro de los editores, Reunión de los Tribunales Constitucionales, y eventos culturales. 
mano (IDH) ${ }^{10}$, Sudáfrica se encontraría en la posición 110 de los 169 países comparados, esto representa el 0,597\%, por lo que Sudáfrica se sitúa por encima de la media regional (Informe de Desarrollo Humano 2011). Brasil en la clasificación de desarrollo humano de las Naciones Unidas tiene un nivel de desarrollo más alto que Sudáfrica y ocupa la posición 73 de los 169 países del ranquin con el 0,699\% (Giaccaglia 2011).

Dentro de Latinoamérica, Brasil está desempeñando un creciente peso geopolítico a nivel regional y a escala mundial, por lo que este país genera influencia dentro y fuera de su propia región. Durante el periodo de Lula da Silva, se incrementaron las relaciones entre Brasil y África en materia de inversiones, intercambio comercial y cultural, permitiendo un acercamiento entre América Latina y el continente africano como modelo de relación Sur-Sur.

\section{Política exterior sudafricana, intereses en Mercosur-UNASUR}

Existen variados factores que incentivan el crecimiento del comercio Sur-Sur, tales como las políticas que favorecen el comercio y la infraestructura del transporte, la rapidez de determinados países en desarrollo en esta área, el fortalecimiento de las redes y transacciones empresariales, interempresariales y sectoriales y el desarrollo de la integración regional, entre otros. Económicamente, observamos que se han incrementado los flujos comerciales internacionales debido a la liberalización y desregulación en los países en vías de desarrollo como factor primordial. En este contexto, el comercio mundial llegó a cuadruplicarse de 2.300 millones de dólares a más de 10.000 millones, entre 1985 y 2005. Así mismo, en estos treinta años, la participación del comercio Sur-Sur en el comercio mundial aumentó del 4,1\% al 11\% (Sousa de 2007: 1-15). A pesar de que el volumen comercial ha aumentado en el marco del IBSA, los acuerdos de libre comercio que se pueden gestar en su ceno tendrán que atravesar un lento camino, ya que tanto Brasil como Sudáfrica presentan diferentes niveles de integración en la economía mundial e integran diferentes mercados regionales. Por un lado, encontramos a Brasil como miembro pleno del Mercado Común del Sur (MERCOSUR) y, por el otro, encontramos a Sudáfrica formando parte del Southern African Customs Union (SACU), ${ }^{11}$ lo cual dificulta el proceso. En la actualidad existe un estancamiento de las relaciones entre el SACU y el MERCOSUR.

10 Desde 1990 el Informe sobre Desarrollo Humano ha publicado el Índice de Desarrollo Humano (IDH) que fue presentado como una alternativa a las mediciones convencionales del desarrollo nacional, como el nivel de ingresos y la tasa de crecimiento económico. El IDH representa el impulso de una definición más amplia del bienestar y ofrece una medida compuesta de tres dimensiones básicas del desarrollo humano: salud, educación e ingresos.

11 La Unión Aduanera del África Meridional surgió el 11 de diciembre de 1969 por un acuerdo entre Sudáfrica, Botswana, Lesotho, Namibia y Swazilandia. La misma entró en vigencia el 1 de marzo de 1970.
La emergencia de los BRICS: Brasil y Sudáfrica en las relaciones Sur-Sur

Carlos Alfredo da Silva 
América Latina: cambios a nivel regional y en su inserción internacional
El 16 de diciembre de 2004, y tras varios años de negociaciones, el MERCOSUR firmó con la SACU un "Acuerdo de Preferencias Arancelarias Fijas" para un conjunto de productos, sobre todo del sector agrícola. Las negociaciones entre ambos bloques tienen como objetivo el establecimiento de una Zona de Libre Comercio. "El problema es la asimetría de las diferentes economías. Pese a que Sudáfrica es un país importante y tiene indicadores comparables a los de Brasil y Argentina, nuestras economías no pueden ser comparadas con las de los otros países africanos y con las de Paraguay y Uruguay", agregó el canciller brasileño (Rousseff 2010).

\section{Conclusiones}

Siguiendo los planteos de la internacionalista brasileña Mónica Hirst (2012: 20-21), el encuentro presidencial Rousseff - Obama, que no fue merecedor del tratamiento de una visita de Estado por parte del protocolo de la Casa Blanca, concentró su atención en temas bilaterales puntuales que, si bien mostraron una nueva divergencia en la relación brasileño - estadounidense, también dejaron en claro la ausencia de sentido estratégico en este vínculo. La pérdida de centralidad de esta relación para Brasil se explica por la transformación reciente de la inserción externa del país en todos los planos. Las relaciones con otros poderes emergentes, como India y Sudáfrica, y con potencias mundiales, como Rusia y China, ofrecen a Brasil un camino fértil de articulaciones y sinergias que contribuye a impulsar el sistema internacional en una dirección multipolar.

Brasil ha intentado actuar como una fuerza de propulsión conducente al mundo multipolar anclado en una multilateralidad reconfigurada. Su empeño es la expansión de las capacidades autónomas y el reconocimiento en un contexto mundial en transición en el que se observa un proceso gradual, desordenado y desigual de difusión del poder internacional. Además de los instrumentos clásicos de poder, tales como el dinamismo económico y dimensión de mercado interno, proyección regional, recursos energéticos y territoriales, el país ha hecho un uso intensivo de sus atributos diplomáticos - profesionales y presidenciales para ampliar su presencia en la escena internacional. Brasil no dispone de recursos de poder duro, muy especialmente la posibilidad de acciones disuasivas que el estatus de potencia atómica asegura. Junto a sus pares del BRICS y del IBSA, busca mostrarse como una nueva fuente de presiones, opiniones y recursos, apoyado en la decisión de ampliar el objetivo de sus responsabilidades y compromisos internacionales.

Un punto crucial es la disociación entre las estrategias global y regional de Brasil ya que su curso de acción no implica la construcción de un liderazgo regional en los ámbitos latino o incluso suramericanos. El lugar de América del Sur en la política externa brasileña ganó relevancia en los años recientes a partir de un esfuerzo redoblado por intensificar su presencia diplomática, el diálogo político, los lazos empresariales, la cooperación para el desarrollo, la 
cooperación militar y policial y el intercambio cultural con todos los países de la región. No obstante, se busca preservar distancia y diferencia entre las piezas y los movimientos observados en los tableros regional y global, evitándose una acumulación lineal de poder que pueda comprometer a uno u otro juego.

La actuación brasileña en cuestiones de política y seguridad internacionales revela una preocupación especial por el tema de la legitimidad del uso de la fuerza en la intervención internacional, como así también el impacto humanitario de la acción militar. Brasil favorece soluciones que procuren un equilibrio entre la paz, la solidaridad, la soberanía y el desarrollo sustentable.

Para Brasil, el año 2011 representó un paso adelante en sus esfuerzos de articulación política con colegas emergentes en el Consejo de Seguridad de la ONU con los cuales se sentó por primera vez de forma simultánea. Las posiciones brasileñas se sumaron a las de sus socios del IBSA, India y Sudáfrica, y, en ocasiones, a las de los socios del BRICS para reforzar posiciones en temas especialmente sensibles como: el reconocimiento del Estado de Palestina, la estabilidad y unidad de Irak, la búsqueda de una solución diplomática con respecto al programa nuclear de Irán, la necesidad de otorgar mayor trascendencia a la Conferencia de Desarme, el proceso de partición de Sudán y la primavera árabe. Por lo planteado anteriormente, creemos necesario observar, en un futuro próximo, cómo Brasil asumirá su rol regional, si pretende la hegemonía en la región o apunta a un liderazgo múltiple (concertado, colaborativo, compartido o distributivo), como lo sostiene el profesor Juan G. Tokatlian (2010: 4).

La noción de países emergentes surgió, especialmente, en función de indicadores de desempeño económicos, su uso está relacionado a un conjunto de Estados empeñados en ampliar su influencia en temas de política y seguridad globales. De esta forma se observa una reconfiguración del rótulo de "economías emergentes" al de "poderes emergentes". No obstante, las pretensiones de Brasil y de sus pares emergentes encuentran aún muchos desafíos y cuestionamientos en cuanto a su naturaleza y posibilidades reales.

Se apunta al riesgo de que el aumento del número de voces pueda significar una alteración cuantitativa, pero no cualitativa, de la agenda y de los métodos acordados en los ámbitos de la gobernabilidad global. Cabe aquí una nueva referencia a la cuestión regional que, en el caso brasileño, brilla por su ausencia en esta edificación. El hecho de que Brasil sea un poder regional no ha significado su proyección como un líder suramericano y menos la articulación entre tal construcción y sus ámbitos globales. El país anhela trazar un plan de ruta - desde un ángulo crítico - que se apoya en la defensa de una gobernanza global reformulada y de construcción de un orden multipolar, lo que se refuerza con la articulación política con otros poderes emergentes por medio de los grupos IBSA y BRICS. También, en este contexto, se comprende que la condición de poder emergente afecta la relación con las potencias industriales, entre las que se destaca Estados Unidos.

Según el antropólogo argentino Néstor García Canclini (2011: 8), “...en América Latina la desigualdad posee bases económicas, históricas, simetrías,
La emergencia de los BRICS: Brasil y Sudáfrica en las relaciones Sur-Sur

Carlos Alfredo da Silva 
América Latina: cambios a nivel regional y en su inserción internacional explotaciones internas y externas. Al mismo tiempo es el resultado de discriminaciones y estereotipos que nos han fijado en el lugar de los que duermen la siesta, de los que no quieren afrontar la complejidad y la dureza del mundo tecnológico, que prefieren las relaciones familiares y de compadrazgo a la competencia económica. Buena parte de la configuración y la reproducción de la desigualdad en Latinoamérica es el resultado de estos estereotipos y de un acceso diferencial y desigual a los recursos de la última modernidad."

Desde que, en 2007, aparecieron los primeros indicios del estallido del capitalismo metropolitano, hasta hoy los presidentes latinoamericanos tuvieron tres tipos de reacciones. Primero aclararon, opulentos, que la región estaba blindada. Poco después se corrigieron: la crisis es importante, pero en América del Sur se ha hecho sentir de manera atenuada. Ahora, sin escapatoria, admiten que la debacle en Europa y Estados Unidos se sentirá en nuestros países aunque seremos los primeros en superarla.

En los hechos, la crisis internacional encontró a los países de la región con holgura fiscal y una situación externa fortalecida por la política de acumulación de reservas. Podríamos afirmar que los países de Latinoamérica y, en especial, los del Sur deberán seguir profundizando sus lazos internos en un contexto internacional donde los "emergentes" lograron alcanzar un crecimiento en el largo plazo. El principal desafío que se les presenta será mejorar la creación de empleo, a través de profundizar las cadenas de valor, la tecnología y valor agregado. El surgimiento de estos bloques (BRICS, UNASUR, CELAC, etc.) tiene que ver con el resurgimiento del Sur, porque cambió la dinámica internacional, convirtiéndose en el motor del crecimiento, consolidando el eje geopolítico Sur-Sur, que no sólo radica en la distribución de la producción, sino que cambia la dinámica del consumo internacional, por una mayor concentración de sectores medios. Como expresa el distinguido filósofo y político brasileño, Roberto Mangabeira Unger, "un verdadero modelo de desarrollo no se puede basar en el voluntarismo político y algunos aciertos económicos; precisa un esfuerzo de construcción institucional y cultura republicana" (Bosoer 2010: 38-39). También considera necesario re-imaginar y reconstruir la economía de mercado y la democracia representativa pensando en una nueva clase media que está surgiendo y en la voluntad de la mayoría pobre. Por ello plantea "directrices a tomar en cuenta: democratizar y extender la economía de mercado con una coordinación estratégica entre gobiernos y empresarios pequeños y medianos, que no es simplemente regularla sino reorganizarla institucionalmente, una política agrícola que asegure atributos a la agricultura familiar, una política laboral hacia los intereses de la mayoría que contemple a la gran cantidad de trabajadores precarizados, temporarios y tercerizados, organizando un estatuto legal para protegerlos y representarlos, cuestionar la pseudo ortodoxia económica de las últimas décadas asegurando un escudo económico para éstas herejías desarrollistas, reafirmando la responsabilidad fiscal, atenuando el recurso a políticas contracíclicas keynesianas. Otra, sumamente relevante una revolución en la educación pública, reconciliando la gestión local de las escuelas con padrones nacionales e inver- 
siones de calidad, ésta última no debe depender del hogar en el que nace el niño, y reorientar radicalmente el paradigma pedagógico sustituyendo el enciclopedismo informativo por una enseñanza analítica capacitadora. La siguiente es sobre el Estado, con tres agendas simultáneas, profesionalismo burocrático, eficiencia administrativa y mejorar los servicios públicos, permitiendo que la sociedad civil participe de la provisión y control, incluida la educación y la salud.

En relación a los desafíos dilemáticos que deberá enfrentar Sudáfrica, se encuentra consecución de una economía más próspera e igualitaria, achicando la brecha entre blancos ricos y negros pobres; quizás lo más frustrante sea el nivel de desempleo que alcanza a un $60 \%$ de los jóvenes negros con baja calificación laboral, representando una eventual amenaza a la joven democracia. Para remediar la situación, parcialmente, deben encararse cambios, postergadas inicialmente. Es urgente la puesta en marcha de reformas en temas cruciales como la propiedad y uso de la tierra, la estructura distorsionada del mercado laboral, la salud, la infraestructura del transporte. El gobierno de coalición de Sudáfrica priorizó la contención política y social sobre las reformas económicas profundas en las últimas décadas, pero esto no ha sido suficiente para completar una exitosa transición política y desarrollo a largo plazo, para asegurar un futuro promisorio.

Reasumiendo, se estaría frente a una etapa de coincidencias en el sur, en especial para Latinoamérica, pero como expresa el distinguido politólogo brasileño, Helio Jaguaribe, existe un peligro agazapado, la "burguesía consular", definiéndola "como una suerte de quinta columna norteamericana que actúan dentro de las sociedades nacionales, vinculados al sistema capitalista dependiente y ligados, directa o indirectamente, a las multinacionales, prefieren la dependencia de los Estados Unidos. Nos quieren convencer que el capital extranjero dará a los países tecnología y progreso, propagando que la dependencia es ventajosa". ${ }^{2}$ Existen ejemplos relevantes en la historia de las relaciones internacionales latinoamericanas de lo antes expresado por Jaguaribe y compartido por distinguidos especialistas de la región. Finalmente, creemos muy importante no olvidar la expresión del ex Secretario de Estado norteamericano, Henry Kissinger, cuando en los años setenta expresó "hacia donde se incline Brasil irá América Latina", pero con referencia a los Estados Unidos de América, como potencia hegemónica, recordaría una sugerencia de O. von Bismarck "hay que ser capaz de advertir cuando lo sólido se transforma en líquido, y lo líquido en sólido".

\section{Bibliografía}

Baumann R. (2009), El comercio entre los países BRICS, CEPAL-ONU.

Bosoer F. (2010), La política argentina debe superar una atracción fatal por tomar siempre atajos, "Clarín", Zona, 25 de julio, pp. 38-39.

12 T. Luzzani, Uno de nuestros peligros es la burguesía consular, "Clarín", Zona, 18 de mayo de 2003, pp. 4-5.
La emergencia de los BRICS: Brasil y Sudáfrica en las relaciones Sur-Sur

Carlos Alfredo da Silva 
América Latina: cambios a nivel regional y en su inserción internacional
Brun E. (2009), Las relaciones entre América Latina y África: ¿potenciales o ilusorias?, CEPAL-ONU.

García Canclini N. (2011), "Revista Tecnología Militar", no 5, Grupo Editorial Mönch, Bonn.

Giaccaglia C. (2011), El Foro de Diálogo Trilateral IBSA: India, Brasil y Sudáfrica, "Otro Sur digital", pp. 1-22.

Gomes Saraiva M. (2010), La política exterior del gobierno de Rousseff, "Revista Umbrales de América del Sur", Prometeo Libros, Buenos Aires, no 12, pp. 69-76.

Grabendorff W. (2010), Brasil: de coloso regional a potencia global, "Nueva Sociedad", marzoabril, no 226, pp. 158-171, disponible en: http://www.nuso.org/upload/articulos/3691_1. pdf

Hirst M. (2012), Los desafíos del Brasil emergente, "Le monde diplomatique", edición especial, Buenos Aires, mayo- junio, pp. 20-21.

Lopes Iole I. (2010), El gobierno de Dilma Rousseff: desafíos y perspectivas, "Revista Umbrales de América del Sur", Prometeo Libros, Buenos Aires, pp. 63-68.

Luzzani T. (2003), Uno de nuestro peligros es la burguesía consular, "Clarín”, Zona, 18 de mayo, pp. 4-5.

O'Neill J. (2001), Building Better Global Economic BRICs, "GS Global Economics Website", Paper, no 66.

Sousa de S. (2008a), Brasil, India y Suráfrica, potencias para un nuevo orden, "Política Exterior", no 121, pp. 1-14.

Sousa de S. (2007), India, Brasil, Sudáfrica (IBSA) ¿Un nuevo tipo de multilateralismo interregional del Sur?, FRIDE, pp. 1-15.

Sousa de S. (2008b), La India, el Brasil y Sudáfrica: ¿potencias emergentes o países en desarrollo?, "El Debate Político", no 36, pp. 1-13

Tokatlian J. G. (2010), El desafío de definir el rol como potencia global, "La Nación”, Enfoques, 6 de noviembre, p. 4.

Wilson D. (2003), Dreaming With BRICs: The Path to 2050, "GS Global Economics Website", no 99.

Zorgbibe Ch. (1997), Historia de las relaciones internacionales. Alianza Editorial, Madrid. 